\title{
SINTOMAS PSICOLÓGICOS EM MULHERES CLIMATÉRICAS CARDIOPATAS
}

\author{
Josilma Silva Nogueira', Bruna da Silva Oliveira², Marli Villela Mamede ${ }^{3}$, \\ Líscia Divana Carvalho Silva ${ }^{4}$
}

\begin{abstract}
RESUMO: Objetivo: investigar os sintomas climatéricos psicológicos em mulheres cardiopatas. Método: estudo quantitativo, realizado em Hospital Universitário no Nordeste do Brasil, no período de outubro de 2016 a janeiro de 2017. Foram entrevistadas individualmente 221 mulheres climatéricas cardiopatas atendidas no Ambulatório de Cardiologia. Foram realizadas análises da estatística descritiva, de associação com o teste não paramétrico de Qui-quadrado de independência $\left(\chi^{2}\right)$ e teste de Correlação de Spearman, com o auxílio do programa SPSS Statistics 20. Resultados: houve predomínio de sintomas climatéricos muito intensos como a angústia/ansiedade, 75 (33,9\%); esgotamento físico e mental, 61 (27,6\%); estado de ânimo depressivo, 54 (24,4\%); nervosismo, 59 (26,7\%); e insônia, 45 (20,4\%). Houve associação significativa nos sintomas climatéricos psicológicos entre si e entre os sintomas psicológicos e a depressão. Conclusão: os sintomas climatéricos psicológicos parecem tornar as mulheres mais propensas a transtornos emocionais, agravada pela existência de uma doença crônica como a cardiopatia.
\end{abstract}

DESCRITORES: Climatério; Menopausa; Cardiopatias; Psicologia; Sintomas psíquicos.

\section{PSYCHOLOGICAL SYMPTOMS IN CLIMACTERIC WOMEN WITH HEART DISEASE}

ABSTRACT: Objective: to investigate psychological climacteric symptoms in women with heart disease. Method: a quantitative study, carried out at a University Hospital in the Northeast of Brazil, from October 2016 to January 2017. A total of 221 climacteric women with heart disease were interviewed at the Cardiology Outpatient Clinic. Descriptive statistical analysis was performed, association with the nonparametric Chi-square test of independence $(\chi 2)$ and Spearman's correlation test, with the use of the SPSS Statistics 20 program. Results: there was a predominance of very intense climacteric symptoms such as anguish/anxiety, 75 (33.9\%); physical and mental exhaustion, 61 (27.6\%); depressed mood, 54 (24.4\%); nervousness, 59 (26.7\%); and insomnia, 45 (20.4\%). There were significant associations among the psychological climacteric symptoms and between the psychological symptoms and depression. Conclusion: psychological climacteric symptoms appear to make women more prone to emotional disorders, aggravated by the existence of a chronic disease such as cardiopathy. KEYWORDS: Climacteric; Menopause; Cardiopathies; Psychology; Psychic symptoms.

\section{SÍNTOMAS PSICOLÓGICOS EN MUJERES EN CLIMATERIO Y CARDIÓPATAS}

RESUMEN: Objetivo: investigar los síntomas climatéricos psicológicos en mujeres cardiópatas. Método: estudio cuantitativo, realizado en Hospital Universitario en Nordeste de Brasil, en el periodo de octubre de 2016 a enero de 2017. Se entrevistaron individualmente 221 mujeres climatéricas cardiópatas atendidas en el Ambulatorio de Cardiología. Se realizaron análisis de estadística descriptiva, de asociación con el test no paramétrico de Chi cuadrado de independencia $\left(\chi^{2}\right)$ y test de Correlación de Spearman, con la ayuda del programa SPSS Statistics 20. Resultados: hubo predominio de síntomas climatéricos muy intensos como angustia/ansiedad, 75 (33,9\%); agotamiento físico y mental, 61 (27,6\%); estado de ánimo depresivo, 54 (24,4\%); nerviosismo, 59 (26,7\%); y insomnio, 45 (20,4\%). Hubo asociación significativa en los síntomas climatéricos psicológicos entre sí y entre los síntomas psicológicos y la depresión. Conclusión: los síntomas climatéricos psicológicos pueden dejar las mujeres más propensas a trastornos emocionales, agraviados por la existencia de una enfermedad crónica como la cardiopatía.

DESCRITORES: Climaterio; Menopausia; Cardiopatías; Psicología; Síntomas psíquicos.

'Enfermeira. Especialista em Clínica Médico-Cirúrgica. Universidade Federal do Maranhão. São Luís-MA-Brasil.

${ }^{2}$ Enfermeira. Mestranda em Enfermagem. Universidade Federal do Maranhão. São Luís-MA-Brasil.

${ }^{3}$ Enfermeira. Doutora em Enfermagem. Docente da Escola de Enfermagem de Ribeirão Preto da Universidade de São Paulo. Ribeirão Preto-SP-Brasil.

${ }^{4}$ Enfermeira. Doutora em Ciências. Docente do Departamento de Enfermagem da Universidade Federal do Maranhão. São Luís-MABrasil. 


\section{INTRODUÇÃO}

O climatério é definido como uma fase biológica da vida da mulher que compreende a transição entre o período reprodutivo e o não reprodutivo, e geralmente ocorre entre os 40 e 65 anos de idade, podendo existir sintomas causados pelo hipoestrogenismo que podem afetar o bem-estar da mulher ${ }^{(1)}$.

Aproximadamente vinte milhões de mulheres estão no período climatérico nos Estados Unidos, com perspectiva de que este número alcance sessenta milhões em 2020. No Brasil, a tendência ao envelhecimento populacional é intensa como registrado nos demais países da América Latina, com estimativa de vida de 78,5 anos, o que enfatiza a importância do climatério/menopausa, trazendo o foco na qualidade de vida como um importante parâmetro de saúde na população feminina ${ }^{(2-3)}$. Observa-se muitas ações voltadas às políticas públicas para o ciclo gravídico-puerperal, para o controle do câncer de colo de útero e de mama. Entretanto, no sentido de concretizar os princípios de integralidade e equidade no sistema de saúde, reconhece-se a necessidade de ampliar a abordagem para outras dimensões, em diferentes momentos do ciclo de vida, como o climatério e a saúde sexual das mulheres ${ }^{(4)}$.

Apesar de o climatério ser uma fase natural da vida, 60 a $80 \%$ das mulheres referem sintomas relacionadas com o hipoestrogenismo. A quantidade e a intensidade da sintomatologia climatérica está relacionada não somente com os níveis hormonais basais de cada mulher, mas aos aspectos étnico, cultural, social, psicológico, afetivo e profissional ${ }^{(5)}$. Essa fase de declínio estrogênico aumenta o risco cardiovascular nas mulheres, constituindo a maior causa de morte em países desenvolvidos e em desenvolvimento ${ }^{(6)}$. Além disso, existe uma preocupação a respeito dos efeitos da terapia de reposição hormonal $(\mathrm{TRH})$ em mulheres climatéricas sobre o risco maior de desenvolver um evento coronariano ${ }^{(7-8)}$.

Acredita-se que mulheres com sintomatologia semelhante percebem de modo diferente essa fase da vida - o climatério e aquelas que compreendem que este é um período fisiológico podem passar por ele como qualquer outra etapa da vida, porém essa situação pode ser agravada fortemente pela presença de uma doença pré-existente, a cardiopatia. O conhecimento sobre a sintomatologia climatérica psicológica pode nortear a busca e seleção de estratégias e ações eficientes, tendo em vista a organização da atenção e integralidade do cuidado na promoção da saúde ao promover reflexões acerca das relações entre dois fenômenos, o climatério e a cardiopatia. Dessa maneira, este estudo tem o objetivo de investigar os sintomas climatéricos psicológicos em mulheres cardiopatas.

\section{MÉTODO}

Estudo descritivo de natureza quantitativa realizado no Ambulatório de Cardiologia do Hospital Universitário da Universidade Federal do Maranhão (HUUFMA), São Luís- MA, Brasil, no período de outubro de 2016 a janeiro de 2017.

Os critérios de inclusão foram mulheres com idades acima de 40 anos, portadoras de cardiopatia e que apresentavam sintomas climatéricos segundo a Escala de Avaliação da Menopausa (MRS)(9). Não foram incluídas mulheres com dificuldades na fala, que apresentavam distúrbios mentais, usuárias de TRH nos últimos cinco anos; e aquelas que não identificaram nenhum sintoma climatérico conforme critérios da MRS. Todas as mulheres identificaram sintomas climatéricos segundo a MRS, portanto, não houve exclusão pela ausência de sintomatologia climatérica.

A MRS é uma escala destinada à avaliação da Qualidade de Vida específica da menopausa. Foi desenvolvida na Alemanha e traduzida e validada em diversos países inclusive no Brasil, que apresentou no teste-reteste um coeficiente de correlação de Pearson de 0,82 e na sua consistência interna através do coeficiente alfa de Cronbach o valor de $0,86^{(10)}$. As mulheres foram interrogadas individualmente enquanto aguardavam a consulta médica, ocasião em que a pesquisadora se apresentava e as convidada a identificar os sintomas psicológicos que elas reconheciam ter experenciado no último ano (12 meses). 
De acordo com a MRS, foi feito o seguinte questionamento: Qual dos seguintes sintomas e em que medida a senhora diria que sentiu nos últimos 12 meses? As participantes também foram investigadas quanto ao histórico de depressão por meio da seguinte questão: "A senhora já teve depressão anteriormente ou tomou remédios para depressão?".

Para análise dos dados, foi utilizado o programa IBM SPSS Statistics 20 (2011). Foram realizadas análises da estatística descritiva das variáveis. A análise da associação das variáveis classificatórias foi feita pelo teste não paramétrico de Qui-quadrado de independência $\left(\chi^{2}\right)$. Foi avaliada a relação dos sintomas psicológicos climatéricos entre si através do teste de Correlação de Spearman. Considerou-se como estatisticamente significante um valor de $\mathrm{p}<0,05$.

Os dados foram coletados a partir do banco de dados da pesquisa intitulada "Mulher climatérica e doença arterial coronariana: desvelando sentidos e significados", realizada no ano de 2013, aprovado pelo Comitê de Ética em Pesquisa da Escola de Enfermagem de Ribeirão Preto da Universidade de São Paulo (EERP-USP), tendo recebido parecer favorável sob o número 293.900.

\section{RESULTADOS}

A amostra do estudo foi constituída por 221 mulheres cardiopatas que apresentavam sintomas climatéricos segundo a Escala MRS. A caracterização sociodemográfica das participantes está descrita abaixo (Tabela 1). Os resultados mostram prevalência de mulheres na faixa etária de 47 a 60 anos, 141 (63,8\%); média etária, 54,8 anos; ensino fundamental completo, 28 (12,7\%); cor branca, 134 (60,6\%); união estável, 108 (48,9\%); e procedentes da capital, 90 (40,7\%). As informações que permaneceram incompletas foram classificadas como ignoradas.

Tabela 1 - Caracterização sociodemográfica das mulheres climatéricas do Hospital Universitário no Nordeste do Brasil. São Luís, MA, Brasil, 2017. (continua)

\begin{tabular}{|c|c|c|}
\hline Variável & $\mathbf{n}$ & $\%$ \\
\hline \multicolumn{3}{|l|}{ Idade } \\
\hline $40-46$ & 30 & 13,6 \\
\hline $47-53$ & 70 & 31,7 \\
\hline $54-60$ & 71 & 32,1 \\
\hline $61-67$ & 47 & 21,2 \\
\hline $68-73$ & 3 & 1,4 \\
\hline \multicolumn{3}{|l|}{$\overline{\text { Cor }}$} \\
\hline Branca & 134 & 60,6 \\
\hline Parda & 62 & 28,1 \\
\hline Negra & 18 & 8,1 \\
\hline Ignorado & 7 & 3,2 \\
\hline \multicolumn{3}{|l|}{ Estado civil } \\
\hline União estável* & 108 & 48,9 \\
\hline Solteiro & 91 & 41,2 \\
\hline Viúva & 7 & 3,2 \\
\hline Divorciada & 5 & 2,3 \\
\hline Ignorado & 10 & 4,5 \\
\hline
\end{tabular}




\begin{tabular}{lcc}
\hline Escolaridade & \multicolumn{2}{c}{} \\
\hline Analfabeto & 26 & 2,7 \\
\hline Ensino Fundamental incompleto & 28 & 11,8 \\
\hline Ensino Fundamental completo & 3 & 12,7 \\
\hline Ensino Médio incompleto & 23 & 1,4 \\
\hline Ensino Médio completo & 1 & 10,4 \\
\hline Ensino Superior incompleto & 3 & 0,5 \\
\hline Ensino Superior completo & 131 & 1,4 \\
\hline Ignorado & \multicolumn{2}{l}{59,3} \\
\hline Procedência & 90 & 40,7 \\
\hline Capital & 74 & 33,5 \\
\hline Outros municípios do Maranhão & 5 & 2,3 \\
\hline Outros estados & 52 & 23,5 \\
\hline Ignorado & 221 & 100 \\
\hline Total & & \\
\hline
\end{tabular}

*Casadas ou que vivem com companheiro

A Tabela 2 refere-se à distribuição das mulheres climatéricas quanto ao tipo de cardiopatia, utilização da TRH há mais de cinco anos e a presença de depressão. A doença coronariana foi prevalente em 68 $(30,8 \%)$ casos, seguida da cardiopatia hipertensiva em 41 (18,5\%) mulheres. Um número significativo de mulheres nega a utilização da TRH 195 (88,2\%) e aquelas que utilizavam, 26 (11,8\%), tinham idades entre 45 e 65 anos. As mulheres que relataram o diagnóstico médico de depressão foram 47 (21,3\%) e destas $25(53,1 \%)$ relataram tratamento com uso de antidepressivos.

Tabela 2 - Distribuição do tipo de cardiopatia, terapia de reposição hormonal e depressão em mulheres climatéricas do Hospital Universitário no Nordeste do Brasil. São Luís, MA, Brasil, 2017

\begin{tabular}{|c|c|c|}
\hline Variável & $\mathbf{n}$ & $\%$ \\
\hline \multicolumn{3}{|l|}{ Cardiopatia } \\
\hline Doença Arterial Coronariana & 68 & 30,8 \\
\hline Cardiopatia Hipertensiva & 41 & 18,5 \\
\hline Arritmia & 27 & 12,2 \\
\hline Valvopatia & 27 & 12,2 \\
\hline Outras & 9 & 4,1 \\
\hline Ignorado & 49 & 22,2 \\
\hline \multicolumn{3}{|l|}{ Terapia de Reposição Hormonal } \\
\hline Não & 195 & 88,2 \\
\hline Sim & 26 & 11,8 \\
\hline \multicolumn{3}{|l|}{ Depressão } \\
\hline Não & 174 & 78,7 \\
\hline Sim & 47 & 21,3 \\
\hline Total & 221 & 100 \\
\hline
\end{tabular}


A Tabela 3 apresenta a graduação da intensidade dos sintomas climatéricos psicológicos, segundo as mulheres cardiopatas. Houve predomínio de sintomas muito intensos como a angústia/ansiedade, 75 (33,9\%); esgotamento físico e mental, 61 (27,6\%); estado de ânimo depressivo, 54 (24,4\%); nervosismo, 59 (26,7\%); e insônia, 45 (20,4\%).

Tabela 3 - Distribuição da intensidade dos sintomas psicológicos em mulheres climatéricas do Hospital Universitário no Nordeste do Brasil. São Luís, MA, Brasil, 2017

\begin{tabular}{|c|c|c|}
\hline Sintomas psicológicos & $\mathbf{n}$ & $\%$ \\
\hline \multicolumn{3}{|l|}{ Insônia } \\
\hline Nenhum & 60 & 27,1 \\
\hline Leve & 33 & 14,9 \\
\hline Moderada & 45 & 20,4 \\
\hline Intensa & 38 & 17,2 \\
\hline Muito intensa & 45 & 20,4 \\
\hline \multicolumn{3}{|l|}{ Estado de ânimo depressivo } \\
\hline Nenhum & 51 & 23,1 \\
\hline Leve & 54 & 24,4 \\
\hline Moderada & 30 & 13,6 \\
\hline Intensa & 32 & 14,5 \\
\hline Muito intensa & 54 & 24,4 \\
\hline \multicolumn{3}{|l|}{ Nervosismo } \\
\hline Nenhum & 35 & 15,8 \\
\hline Leve & 45 & 20,4 \\
\hline Moderada & 40 & 18,1 \\
\hline Intensa & 42 & 19 \\
\hline Muito intensa & 59 & 26,7 \\
\hline \multicolumn{3}{|l|}{ Angústia/Ansiedade } \\
\hline Nenhum & 32 & 14,5 \\
\hline Leve & 33 & 14,9 \\
\hline Moderada & 49 & 22,2 \\
\hline Intensa & 32 & 14,5 \\
\hline Muito intensa & 75 & 33,9 \\
\hline \multicolumn{3}{|l|}{ Esgotamento físico e mental } \\
\hline Nenhum & 33 & 14,9 \\
\hline Leve & 37 & 16,7 \\
\hline Moderada & 45 & 20,4 \\
\hline Intensa & 45 & 20,4 \\
\hline Muito intensa & 61 & 27,6 \\
\hline Total & 221 & 100 \\
\hline
\end{tabular}

Os Quadros 1 e 2 referem-se, respectivamente, à associação dos sintomas climatéricos psicológicos com as variáveis do estudo e a correlação dos sintomas climatéricos psicológicos entre si. 
Quadro 1 - Associação dos sintomas psicológicos em relação às variáveis sociodemográficas e clínicas em mulheres climatéricas do Hospital Universitário no Nordeste do Brasil. São Luís, MA, Brasil, 2017

\begin{tabular}{lcccccccc}
\hline Sintomas psicológicos & Idade & Cor & Escolaridade & Estado civil & Procedência & THR $^{*}$ & Patologia & Depressão \\
\hline Insônia & 0,228 & 0,380 & 0,112 & 0,483 & 0,810 & 0,400 & 0,898 & 0,057 \\
\hline $\begin{array}{l}\text { Estado de ânimo } \\
\text { depressivo }\end{array}$ & 0,406 & 0,843 & 0,380 & 0,583 & 0,701 & 0,127 & 0,433 & 0,000 \\
\hline Nervosismo & 0,569 & 0,746 & 0,799 & 0,491 & 0,589 & 0,269 & 0,634 & 0,007 \\
\hline $\begin{array}{l}\text { Angústia/ } \\
\text { Ansiedade }\end{array}$ & 0,266 & 0,747 & 0,874 & 0,870 & 0,081 & 0,211 & 0,590 & 0,002 \\
\hline $\begin{array}{l}\text { Esgotamento físico e } \\
\text { mental }\end{array}$ & 0,408 & 0,762 & 0,594 & 0,787 & 0,729 & 0,810 & 0,432 & 0,000 \\
\hline
\end{tabular}

Teste de Qui-quadrado * Terapia de Reposição Hormonal

Quadro 2 - Correlação entre os sintomas psicológicos em mulheres climatéricas do Hospital Universitário no Nordeste do Brasil. São Luís, MA, Brasil, 2017

\begin{tabular}{lccccc}
\hline & Insônia & $\begin{array}{c}\text { Estado de ânimo } \\
\text { depressivo }\end{array}$ & Nervosismo & $\begin{array}{c}\text { Angústia/ } \\
\text { Ansiedade }\end{array}$ & $\begin{array}{c}\text { Esgotamento físico } \\
\text { e mental }\end{array}$ \\
\hline Insônia & & $0,387^{*}$ & $0,402^{*}$ & $0,369^{*}$ & $0,399^{*}$ \\
\hline Estado de ânimo depressivo & $0,387^{*}$ & & $0,613^{*}$ & $0,583^{*}$ & $0,452^{*}$ \\
\hline Nervosismo & $0,402^{*}$ & $0,613^{*}$ & & $0,722^{*}$ & $0,511^{*}$ \\
\hline Angústia/ Ansiedade & $0,369^{*}$ & $0,583^{*}$ & $0,722^{*}$ & & $0,430^{*}$ \\
\hline Esgotamento físico e mental & $0,399^{*}$ & $0,452^{*}$ & $0,511^{*}$ & $0,430^{*}$ & \\
\hline
\end{tabular}

Teste de Correlação de Spearman

* Significa $p<0,05$

Identifica-se uma associação significativa entre os sintomas climatéricos estado de ânimo depressivo $\left(p_{=} 0,000\right)$, nervosismo $(p=0,007)$, angústia/ansiedade $(p=0,002)$ e esgotamento físico e mental $(p=$ 0,000 ) e a variável de depressão (Quadro 1). O nível de significância para se rejeitar a hipótese de nulidade foi de 5\%, ou seja, considerou-se como estatisticamente significante um valor de $p<0,05$. Foi encontrada uma correlação positiva entre os sintomas climatéricos psicológicos, indicando que o aumento da intensidade de um sintoma fazia com que a intensidade de outro sintoma aumentasse também (Quadro 2).

\section{DISCUSSÃO}

Existem poucos estudos de base populacional, nacionais ou latino-americanos sobre a epidemiologia do climatério e da menopausa. A maioria dos dados disponíveis origina-se de países desenvolvidos da América do Norte e Europa. A escassez de trabalhos demanda uma melhor avaliação das mulheres brasileiras ${ }^{(11-12)}$.

O período em que a mulher poderá vivenciar os sintomas climatéricos comumente é a partir dos 40 anos. Neste estudo a média etária foi de 54,8 anos. Pesquisas em mulheres climatéricas apontam para uma média etária entre 43,8 e 54 anos nos países em desenvolvimento, inferior à encontrada em países desenvolvidos que ocorre de 49,9 a 58,7 $\operatorname{anos}^{(12)}$. Fato que tem trazido discussões acerca das repercussões da menopausa para as mulheres em diferentes populações e grupos étnicos, devido ao acelerado processo de envelhecimento populacional e a vulnerabilidade social e econômica desses países. O prolongamento da expectativa de vida feminina gerou um interesse crescente acerca do climatério e suas implicações para a saúde da mulher, por ser um período que é influenciado tanto 
por fatores biológicos relacionados com a redução dos níveis estrogênicos, como por fatores sociais, econômicos, culturais e psicológicos relacionados com o processo de envelhecimento ${ }^{(6,11)}$.

A escolaridade pode ser fundamental para o conhecimento e compreensão sobre as mudanças advindas do climatério, reduzindo mitos e preconceitos relacionados com essa fase. ${ }^{(13)} \mathrm{O}$ nível de escolaridade deste estudo foi considerado baixo, o que determina um impacto significativo na compreensão e percepção do envelhecimento, do climatério, de uma doença pré-existente e do estado de saúde. Considera-se a escolaridade, dentre os indicadores socioeconômicos, o preditor mais potente de fator de risco coronariano, cujo impacto independe da renda sobre esses fatores. Na mulher climatérica cardiopata, a escolaridade é uma variável importante para a sua condição de saúde, revela-se como um pré-requisito para o autocuidado, motivação e alcance de metas que levem à adesão ao tratamento ${ }^{(14)}$.

Sabe-se que a doença coronariana nas mulheres se deve essencialmente à perda do efeito vasodilatador e protetor do estrogênio, tanto ao nível sistêmico como regional que parece ser dependente do endotélio ${ }^{(15)}$. Além disso, a mulher no climatério sofre alterações adversas na composição da gordura corporal (lipídios e lipoproteínas) e no remodelamento vascular; como consequência, aumenta o risco para uma doença cardíaca ${ }^{(16)}$. Atualmente a TRH é indicada somente como medida terapêutica para o alívio dos sintomas climatéricos moderados a intensos, gerando benefícios sobre a qualidade de vida, o que talvez explique o número inexpressivo de mulheres no presente estudo que a utilizou $26(11,8 \%)$.

Reconhece-se que as mulheres respondem de forma diferente à terapia hormonal, especialmente no que diz respeito à idade da menopausa, ao tempo de diagnóstico da doença cardíaca e ao início e término da terapia. Há efeitos benéficos da terapia quanto à morbimortalidade coronariana quando iniciada em mulheres mais jovens $(<60$ anos) e perto da menopausa $(<10$ anos desde a menopausa). Por outro lado, parece haver efeito nulo e possivelmente adverso quando iniciada em mulheres mais velhas ( $\geq 60$ anos) ou com muito tempo de menopausa (> 20 anos) $)^{(17)}$.

Estudos observacionais e randomizados concluem que as mulheres que iniciam a TRH após 10 anos de menopausa apresentam risco aumentado de evento cardiovascular, ao contrário daquelas que iniciam a terapia de maneira precoce, apresentando um risco baixo, além de responderem de forma variada. Recomenda-se individualizar a TRH, tratando as mulheres com a menor dose efetiva e pelo menor tempo possível ${ }^{(6-7)}$.

No presente estudo não foi encontrada nenhuma associação em relação aos sintomas psicológicos e à realização da TRH. Entretanto, houve uma correlação positiva entre os próprios sintomas psicológicos e a presença de depressão.

Os sintomas de depressão no climatério ocorrem frequentemente na forma de distúrbios somáticos, tais como: angina, distúrbios do trato gastrointestinal, síndrome de pernas inquietas, prurido, dores de cabeça, nas costas, e nas articulações ${ }^{(12,18)}$. A depressão é considerada um fator de risco para cardiopatia em mulheres climatéricas. Os consensos para tratamento de depressão indicam que medicação e intervenção comportamental são comumente efetivas e que a combinação das mesmas diminui a taxa de recorrência da doença cardíaca. Não há evidência disponível que demonstre ser diferente o tratamento de depressão em pessoas cardiopatas, porém, existe um risco aumentado de eventos cardiovasculares adversos naquelas em tratamento para depressão ${ }^{(19)}$.

Nessa perspectiva, há necessidade de uma maior interação entre a população de mulheres climatéricas, ginecologistas e psiquiatras/psicólogos, a fim de promover a detecção e a oferta de tratamento de transtornos mentais àquelas sintomáticas, cujos sintomas são altamente comprometedores da qualidade de vida. Aponta-se para uma relação significativa entre a presença de sintomas depressivos de intensidade moderada e as alterações do humor, o que pode ser resultado de vários fatores, como as flutuações hormonais que ocorrem nessa fase, os aspectos sociais e emocionais como mudanças no meio familiar, a separação conjugal, a síndrome do ninho vazio, a doença ou morte de familiares, a diminuição de renda, a aposentadoria, dentre outros. Além disso, sobrepõe-se a dificuldade que têm as mulheres de procurar atendimento psicológico/psiquiátrico para transtornos predominantemente leves e moderados, diante do estigma que a especialidade ainda carrega ${ }^{(20)}$. 
No presente estudo, a frequência dos sintomas climatéricos psicológicos de intensidade moderada a muito intensa foi prevalente e parece existir uma correlação entre os sintomas psicológicos e físicos que potencializam a manifestação climatérica. Acredita-se que sintomas físicos como as ondas de calor são considerados como causa comum de insônia durante o climatério, sendo responsáveis pelo aumento de episódios de despertar noturnos e pela redução do sono, uma vez que os calores e a sudorese noturna têm sido associados como causa para as alterações de sono ${ }^{(12,21-22)}$.

A irritabilidade, o baixo nível de escolaridade e a perda de memória podem ser considerados como fatores de risco para transtornos psiquiátricos leves, em mulheres em transição de menopausa submetidas a uma triagem ambulatorial, seguida por acompanhamento domiciliar. A irritabilidade pode se manifestar na presença de insônia, ansiedade e queixas vasomotoras ${ }^{(23)}$. A própria diminuição significativa estrogênica, além de levar à ansiedade, pode se manifestar pela presença da irritabilidade, tensão muscular, inquietação, taquicardia, sudorese, fadiga e preocupação excessiva com problemas. A menopausa aliada a uma condição socioeconômica desfavorável exerce uma influência direta no determinismo do aparecimento da ansiedade ${ }^{(11)}$.

Estudo multicêntrico realizado nos Estados Unidos encontrou associação significativa entre a menopausa precoce e a ocorrência de fadiga e intolerância a atividades ${ }^{(22)}$. Estudos nacionais têm demonstrado que o esgotamento físico e mental está presente nas mulheres em todas as fases de transição menopáusica. A fadiga tem sido relacionada com piora da qualidade de vida de mulheres cardiopatas, pois a fisiopatologia da própria doença cardíaca traz uma limitação física, dificultando que estas mulheres realizem suas atividades e afazeres do cotidiano. Desta forma, compromete sua autonomia e gera impacto negativo, tanto nos seus hábitos de vida como na sua relação na dinâmica familiar ${ }^{(24)}$.

A sintomatologia pode ocorrer sob influência de diversos fatores, o que pode impactar positivamente ou negativamente nas atividades exercidas, no cotidiano das mulheres, nas suas relações profissionais, familiares e sociais. Em contrapartida, mulheres podem apresentar maior habilidade de adaptação, descobrindo novas atividades, realizando planos e desempenhando novos papéis ocupacionais. O preconceito social e o desconhecimento pela mulher sobre as mudanças ocorridas nesse período se constituem em barreiras, afetando os cuidados necessários com a saúde e, consequentemente, prejudicando sua qualidade de vida ${ }^{(25)}$.

Ressalta-se, pois, que o climatério é uma fase da vida que torna as mulheres mais propensas a sintomas como irritabilidade, nervosismo e frequentes alterações no humor, sendo os sintomas mais intensos naquelas mulheres portadoras de doenças crônicas ${ }^{(11)}$. Durante a menopausa, sintomas psicológicos podem causar um impacto significativo na qualidade de vida e capacidade para o trabalho das mulheres, o que favorece a suscetibilidade à depressão e ao aparecimento de outros sintomas, sendo mais agravado com uma doença cardíaca.

Em razão de muitas mulheres apresentarem humor deprimido devido à menopausa, muitas são diagnosticadas com depressão. Um estudo que avaliou a qualidade de vida em mulheres no climatério, atendidas na atenção primária, mostrou um número significativo de mulheres que faziam uso de tranquilizantes por queixas climatéricas. No entanto, não era feita a correlação de que essa manifestação se dava pelo climatério, mas sim pela presença de depressão, o que poderia representar em alguns casos um erro de diagnóstico ${ }^{(22,26)}$. Estudos longitudinais mostraram que mulheres com sintomas psicológicos intensos, transtornos emocionais ou depressão, são mais propensas a ter eventos coronarianos. Entretanto, não se sabe até que ponto os sintomas psicologicos são influenciados pela doença cardíada ou se a doença cardíaca interferiu na sintomatologia climatérica ${ }^{(27)}$.

A ansiedade está ligada a sentimentos de medo, de tensão e de perigo, sendo desencadeada no contato com algo novo ou em situações inesperadas ou desconhecidas, como é o caso do climatério, que para muitas mulheres é uma fase desconhecida. Os sintomas são os mais diversos: taquicardia, tremores, tensão muscular, aumento do desejo de urinar e defecar, cefaleia, epigastralgia, entre outros. Esta manifestação está associada a piores resultados em qualidade de vida, especialmente nas dimensões psicológica e social, em pacientes com doenças crônicas. Desta forma, questiona-se se a ansiedade é 
causa ou consequência das doenças, pois a relação entre os sintomas psicológicos e as doenças cardíacas tem sido considerada bidirecional, ou seja, situações de estresse e ansiedade podem ter influência no desencadear da cardiopatia; assim como a cardiopatia pode gerar estresse e sintomas de ansiedade ${ }^{(28)}$.

Tem-se encontrado associação entre sintomas psicológicos e as doenças, ou seja, as emoções podem influenciar o aparecimento de doenças específicas ou podem ser consequência de doenças, influenciando seu prognóstico. A relação entre emoções e a saúde cardiovascular é, então, bidirecional, tendo seu efeito cumulativo no decorrer do tempo. A ansiedade, por exemplo, para além de sintoma climatérico, é comum na doença cardíaca e está independentemente relacionada com o aumento da morbimortalidade, principalmente quando associada à presença da depressão(29).

Pressupõe-se que as mudanças das mulheres advindas do envelhecimento e do climatério geram sentimentos como angústia, ansiedade e insegurança que, somadas à vivência com a cardiopatia, produzem sensação de medo, finitude e ameaça a sua vida. As experiências com uma realidade completamente diferente, a convivência com o climatério e uma doença estigmatizante, promove sentimentos muitas vezes negativos de enfrentamento.

Considera-se como limitações do estudo a ausência de algumas informações ginecológicas, obstétricas e o estágio do climatério o que poderia contribuir para uma melhor acurácia na análise dos dados.

\section{CONCLUSÕES}

Os sintomas climatéricos psicológicos, identificados como angústia, ansiedade, esgotamento físico e mental, estado de ânimo depressivo, nervosismo e insônia, parecem tornar as mulheres mais propensas a transtornos emocionais. Acresce-se o fato de que a cardiopatia se apresenta como uma das principais causas para o desenvolvimento de sintomas psicológicos, pois a doença crônica está constantemente associada a um aumento na prevalência de sintomas depressivos e da depressão em si.

Observa-se uma complexa associação entre esses dois fenômenos, climatério e doença cardíaca, devendo-se, por isso, ser mais estudada e compreendida, constituindo-se uma prioridade na Saúde Pública e gerando subsídios para um cuidado integral. Compreender a sintomatologia climatérica em mulheres cardiopatas poderá fundamentar estratégias terapêuticas mais eficazes de atenção no processo de envelhecimento, no climatério e na cardiologia, sendo essencial para uma vivência mais saudável da mulher nessa fase da vida (climatério) e, consequentemente, na vivência de uma doença tão estigmatizante (cardiopatia).

\section{REFERÊNCIAS}

1. Gallon CW, Wender COM. Estado nutricional e qualidade de vida da mulher climatérica. Rev. Bras. Ginecol. Obstet. [Internet] 2012;34(4) [acesso em 01 fev 2017]. Disponível: http://dx.doi.org/10.1590/S0100-72032012000400007.

2. Gold EB, Crawford SL, Avis NE, Crandall CJ, Matthews KA, Waetjen LE, et al. Factors related to age at natural menopause: longitudinal analyses from SWAN. American J. of Epidemiology. [Internet] 2013;178(1) [acesso em 14 fev 2017]. Disponível: https://doi.org/10.1093/aje/kws421.

3. Instituto Brasileiro de Geografia e Estatística (IBGE). Ministério do Planejamento, Orçamento e Gestão. Síntese de indicadores sociais: uma análise das condições de vida da população brasileira - 2014. [Internet] Rio de Janeiro: IBGE; 2014 [acesso em 01 fev 2017]. Disponível: http://biblioteca.ibge.gov.br/visualizacao/livros/liv91983.pdf.

4. Ministério da Saúde (BR). Secretaria de Atenção à Saúde. Departamento de Atenção Básica. Saúde sexual e saúde reprodutiva (Cadernos de Atenção Básica; 26). [Internet] Brasília: Ministério da Saúde; 2013 [acesso em 10 mar 2017 ]. Disponível: http://bvsms.saude.gov.br/bvs/publicacoes/saude_sexual_saude_reprodutiva.pdf. 
5. Peixoto LN, Araujo MFS, Egydio CA, Ribeiro FE, Fregonesi CEPT, do Carmo EM. Perfil e intensidade de sintomas de mulheres no climatério avaliadas em unidades básicas de saúde de presidente prudente. Colloquium Vitae. [Internet] 2015;07(1) [acesso em 10 mar 2017]. Disponível: http://dx.doi.org/ 10.5747/cv.2015.v07.n1.v129.

6. Lobo RA, Davis SR, De Villiers TJ, Gompel A, Henderson VW, Hodis HN, et al. Prevenção de doenças após a menopausa. 2014. International Menopause Society. Climacteric. [Internet] 2014;17 [acesso em 06 mar 2017]. Disponível:

http://www.imsociety.org/manage/images/pdf/19626fd1bff08acf1ca8ed3490e8f002.pdf.

7. Associação Brasileira de Climatério (SOBRAC). Consenso Brasileiro de Terapêutica Hormonal da Menopausa. [Internet] São Paulo: SOBRAC; 2014 [acesso em 02 fev 2017]. Disponível: http://sobrac.org.br/publicacoes/artigos\#Sobrac.

8. Soares Júnior JM, Sorpreso ICE, Baracat EC. Is hormone therapy during climacteric for all? Rev. Assoc. Med. Bras. [Internet] 2015;61(3) [acesso em 12 mar 2017]. Disponível: http://dx.doi.org/10.1590/1806-9282.61.03.191.

9. Heinemann LA, Ruebig A, Potthoff P, Schneider HPG, Strelow F, Heinemann LAJ, et al. The Menopause Rating Scale (MRS): A methodological review. Health and Quality of Life Outcomes. [Internet] 2004;2(45) [acesso em 06 mar 2017]. Disponível: http://dx.doi.org/ 10.1186/1477-7525-2-45.

10. Esper EMB. O climatério na contemporaneidade [dissertação]. São Paulo (SP): Pontifícia Universidade Católica de São Paulo; 2005.

11. Filho JFL, Baccaro LFC, Fernandes T, Conde DM, Costa-Paiva L, Neto AMP. Epidemiologia da menopausa e dos sintomas climatéricos em mulheres de uma região metropolitana no sudeste do Brasil: inquérito populacional domiciliar. Rev. Bras. Ginecol. Obstet. [Internet] 2015;37(4) [acesso em 05 fev 2017]. Disponível: http://dx.doi.org/10.1590/SO100720320150005282.

12. dos Santos TR, Pereira SVM, Santos RL. Intensidade da sintomatologia climatérica em mulheres pós-menopausa. Rev. Rene. [Internet] 2016;17(2) [acesso em 05 fev 2017]. Disponível: http://dx.doi.org/10.15253/2175-6783.2016000200010.

13. Ribeiro AS, Soares AKA, Siqueira VMS, Souza WA, Podestá MHMC, Ferreira EB. Avaliação dos sintomas e da qualidade de vida das mulheres no climatério. Rev. Universidade Vale do Rio Verde. [Internet] 2015;13(1) [acesso em 06 mar 2017]. Disponível: http: http://periodicos.unincor.br/index.php/revistaunincor/article/view/1837/pdf_289.

14. Martin RDS, Godoy ID, Franco RJDS, Martin LC, Martins AS. Influência do nível socioeconômico sobre os fatores de risco cardiovascular. J. Bras. Med. [Internet] 2014;102(20) [acesso em 08 fev 2017]. Disponível: http://files.bvs.br/ upload/S/0047-2077/2014/v102n2/a4193.pdf.

15. Simão AF, Précoma DB, de Andrade JP, Filho HC, Saraiva JFK, de Oliveira LMM. I Cardiovascular Prevention Guideline of the Brazilian Society of Cardiology - Executive Summary. Arq. Bras. Cardiol. [Internet] 2014;102(5) [acesso em 01 out 2016]. Disponível: http://dx.doi.org/10.5935/abc.20140067.

16. El Khoudary SR, Shields KJ, Janssen I, Budoff MJ, Everson-Rose SA, Powell LH, et al. Postmenopausal Women with Greater Paracardial Fat Have More Coronary Artery Calcification Than Premenopausal Women: The Study of Women's Health across the Nation (SWAN) Cardiovascular Fat Ancillary Study. J. Am. Heart Assoc. [Internet] 2017;6(2) [acesso em 06 mar 2017]. Disponível: https://doi.org/10.1161/JAHA.116.004545.

17. Hodis HN, Mack WJ. The Timing Hypothesis and Hormone Replacement Therapy: a Paradigm Shift in the Primary Prevention of Coronary Heart Disease in Women. Part 2: Comparative Risks. J. Am. Geriatr. Soc. [Internet] 2013;61(6) [acesso em 08 mar 2017]. Disponível: https://doi.org/10.1111/jgs.12281.

18. Rauma PH, Koivumaa-Honkanen H, Kröger $\mathrm{H}$, Tuppurainen MT, Kauhanen J, Honkanen RJ. The Relationship Between self-reported and registry-based data on use of psychoactive medications in postmenopausal women. BMC Psychiatry. [Internet] 2013;13(180) [acesso em 06 mar 2017]. Disponível: https://doi.org/10.1186/1471-244X-13-180.

19. Barrett-Connor E. Menopause, atherosclerosis, and coronary artery disease. Curr. Opin. Pharmacol. [Internet] 2013;13(2) [acesso em 08 mar 2017]. Disponível: https://doi.org/10.1016/j.coph.2013.01.005. 
20. Polisseni AF, Araujo DAC, Polisseni F, Júnior CAM, Polisseni J, Fernandes ES, et al. Depressão e ansiedade em mulheres climatéricas: fatores associados. Rev. Bras. Ginecol. Obstet. [Internet] 2009;31(1) [acesso em 01 fev 2017]. Disponível: http: http://dx.doi.org/10.1590/S0100-72032009000100006.

21. Freitas RF, Freitas TF, Pinto PAF, Vieira DR, Pereira SC, Royo VA, et al. Contribuição do profissional farmacêutico na melhoria da qualidade de vida de mulheres climatéricas. Rev. Eletr. Gestão e Saúde. [Internet] 2015;6(2) [acesso em 10 mar 2017]. Disponível: http://dx.doi.org/10.18673/gs.v6i2.22508.

22. Hess R, Thurston RC, Hays RD, Chang CCH, Dillon SN, Ness RB, et al. The impact of menopause on health-related quality of life: results from the STRIDE longitudinal study. Qual. Life Res. [Internet] 2012;21(3) [acesso em 20 fev 2017]. Disponível: http://dx.doi.org/10.1007/s11136-011-9959-7.

23. Araújo JBS, Santos GC, Nascimento MA, Dantas JGS, Ribeiro ASC. Avaliação da intensidade da sintomatologia do climatério em mulheres: Inquérito populacional na cidade de Maceió, Alagoas. Ciênc. Biol. Saúde. [Internet] 2015;2(3) [acesso em 06 mar 2017]. Disponível: https://periodicos.set.edu.br/index.php/fitsbiosaude/article/view/2092/1269.

24. Staniute M, Bunevicius A, Brozaitiene J, Bunevicius R. Relationship of health-related quality of life with fatigue and exercise capacity in patients with coronary artery disease. Eur. J. Cardiovasc. Nurs. [Internet] 2014;13(4) [acesso em 20 fev 2017]. Disponível: http://dx.doi.org/10.1177/1474515113496942.

25. Cardoso MR, de Camargo MJG. Percepções sobre as mudanças nas atividades cotidianas e nos papéis ocupacionais de mulheres no climatério. Cad. Ter. Ocup. UFSCar. [Internet] 2015;23(3) [acesso em 06 mar 2017]. Disponível: https:// doi.org/10.4322/0104-4931.ctoAO0574.

26. Miranda JS, Ferreira MLSM, Corrente JE. Qualidade de vida em mulheres no climatério atendidas na Atenção Primária. Rev. bras. enferm. [Internet] 2014;67(5) [acesso em 07 fev 2017]. Disponível: http://dx.doi.org/10.1590/00347167.2014670519.

27. Llaneza P, García-Portilla MP, Llaneza-Suárez D, Armott B, Pérez-López FR. Depressive disorders and the menopause transition. Maturitas. [Internet] 2012;71(2) [acesso em 14 mar 2017]. Disponível: http://dx.doi.org/10.1016/j. maturitas.2011.11.017.

28. França AB. Sintomas depressivos e ansiosos em pacientes com doença isquêmica do coração e variáveis associadas [dissertação]. Florianópolis (SC): Universidade Federal de Santa Catarina; 2013.

29. Hare DL, Toukhsati SR, Johansson P, Jaarsma T. Depression and cardiovascular disease: a clinical review. Eur. Heart J. [Internet] 2014;35(21) [acesso em 01 fev 2017]. Disponível: https://doi.org/10.1093/eurheartj/eht462. 